Revue des patrimoines

\title{
Des vitraux civils bretons conservés au Québec
}

\section{Françoise Gatouillat}

\section{OpenEdition}

Journals

Édition électronique

URL : http://journals.openedition.org/insitu/2070

DOI : 10.4000/insitu.2070

ISSN : $1630-7305$

\section{Éditeur}

Ministère de la culture

\section{Référence électronique}

Françoise Gatouillat, «Des vitraux civils bretons conservés au Québec », In Situ [En ligne], 3 | 2003, mis en ligne le 23 avril 2012, consulté le 01 mai 2019. URL : http://journals.openedition.org/insitu/2070 ; DOI : $10.4000 /$ insitu. 2070

Ce document a été généré automatiquement le 1 mai 2019.

\section{(c)}

In Situ Revues des patrimoines est mis à disposition selon les termes de la licence Creative Commons Attribution - Pas d'Utilisation Commerciale - Pas de Modification 4.0 International. 


\title{
Des vitraux civils bretons conservés au Québec
}

\author{
Françoise Gatouillat
}

1 Les verrières sont des objets mobiles; la collection du Recensement des vitraux anciens de la France ${ }^{1}$ en fait état dans ses listes annexes, alimentées par des enquêtes qui aboutissent parfois à des découvertes inespérées. L'inventaire des vitraux de Bretagne est près de s'achever. Afin de compléter la rubrique consacrée aux vitraux erratiques de la région, contact a été pris avec le responsable du comité canadien du Corpus Vitrearum, M. Roland Sanfaçon, pour tenter de localiser ceux qui ornaient autrefois le manoir de Limoëlou dit aussi des Portes-Cartier à Paramé, commune de Saint-Malo, où résida Jacques Cartier jusqu'à sa mort en 1557. Dans sa statistique monumentale d'Ille-etVilaine, Paul Banéat avait signalé en 1929 l'existence de ces vitraux, précisant que deux panneaux, parmi lesquels une figure de saint Julien, avaient été offerts au Québec en souvenir du navigateur². Grâce à son efficace réseau savant, la recherche de M. Sanfaçon devait bientôt aboutir : le vitrail de saint Julien se trouve conservé au musée du Séminaire de Sherbrooke et son jumeau, dont on ignorait tout, fait partie des collections du musée du Château Ramezay à Montréal. Les photographies d'ensemble et de détails prises par l'éminent spécialiste, ses observations scientifiques ainsi que les informations que livraient les pièces d'archives qui lui ont été communiquées nous ont aussitôt été transmises avec la plus grande générosité ${ }^{3}$. Les oeuvres en question qui, à la lecture du texte de 1929, s'annonçaient comme des «rondels» d'un type assez commun, devaient réserver une agréable surprise. Les panneaux retrouvés au Canada sont des châssis vitrés complets de fenêtres civiles du XVIIe siècle, avec leur cadre de bois et des éléments de leur serrurerie d'origine, dont on ne connaît plus guère d'exemple en France. De plus, le parti ornemental adopté se distingue de la formule qui, en matière de vitrage domestique, était la plus répandue, à savoir des petits sujets peints sur une pièce de verre unique enchâssée dans des losanges ou des bornes incolores. Ce sont donc des objets d'un intérêt exceptionnel. 
Figure 1

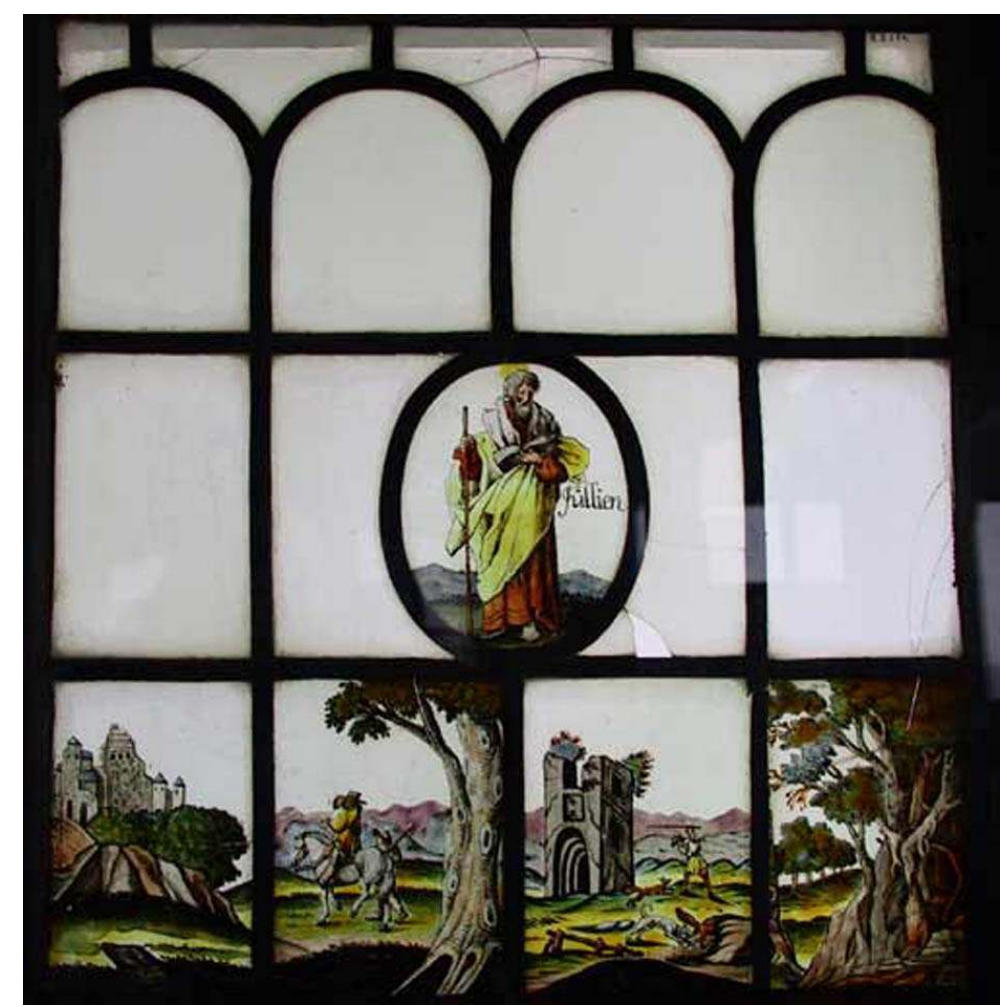

Musée du Séminaire de Sherbrooke (Québec) : châssis vitré provenant du manoir de Limoëlou à Paramé, vue d'ensemble

(c) R. Sanfaçon, 2002

2 Chacun des panneaux, de même dimension -environ $54 \mathrm{~cm}$ de hauteur sur 50 de large-, est constitué d'un assemblage de pièces blanches et de pièces peintes mises en plombs, agencées de manière régulière. Le centre en est occupé par un ovale représentant un saint debout, identifié par une inscription, «Jullien » (sic) dans le vitrail de Sherbrooke et Bertrand dans celui de Montréal, deux proto-évêques du Mans dont l'image fut manifestement inspirée ici par quelque suite d'apôtres gravée. La vitrerie d'accompagnement, échancrée autour des rondels, est faite de pièces rectilignes dans les deux rangs inférieurs, sous un rang de pièces cintrées; celles-ci s'imbriquent à la limite supérieure de la composition dans d'autres, plus petites et de coupe appropriée, signe du soin particulier apporté à la conception et à la réalisation de ces oeuvres ${ }^{4}$. Grande originalité, le décor peint ne se limite pas à l'ovale hagiographique mais s'étend à l'ensemble des carreaux de la zone inférieure. Un paysage vallonné, animé de petits personnages évoluant devant des fabriques, s'y développe en une frise rythmée de grands arbres placés à l'avant-plan. 
Figure 2

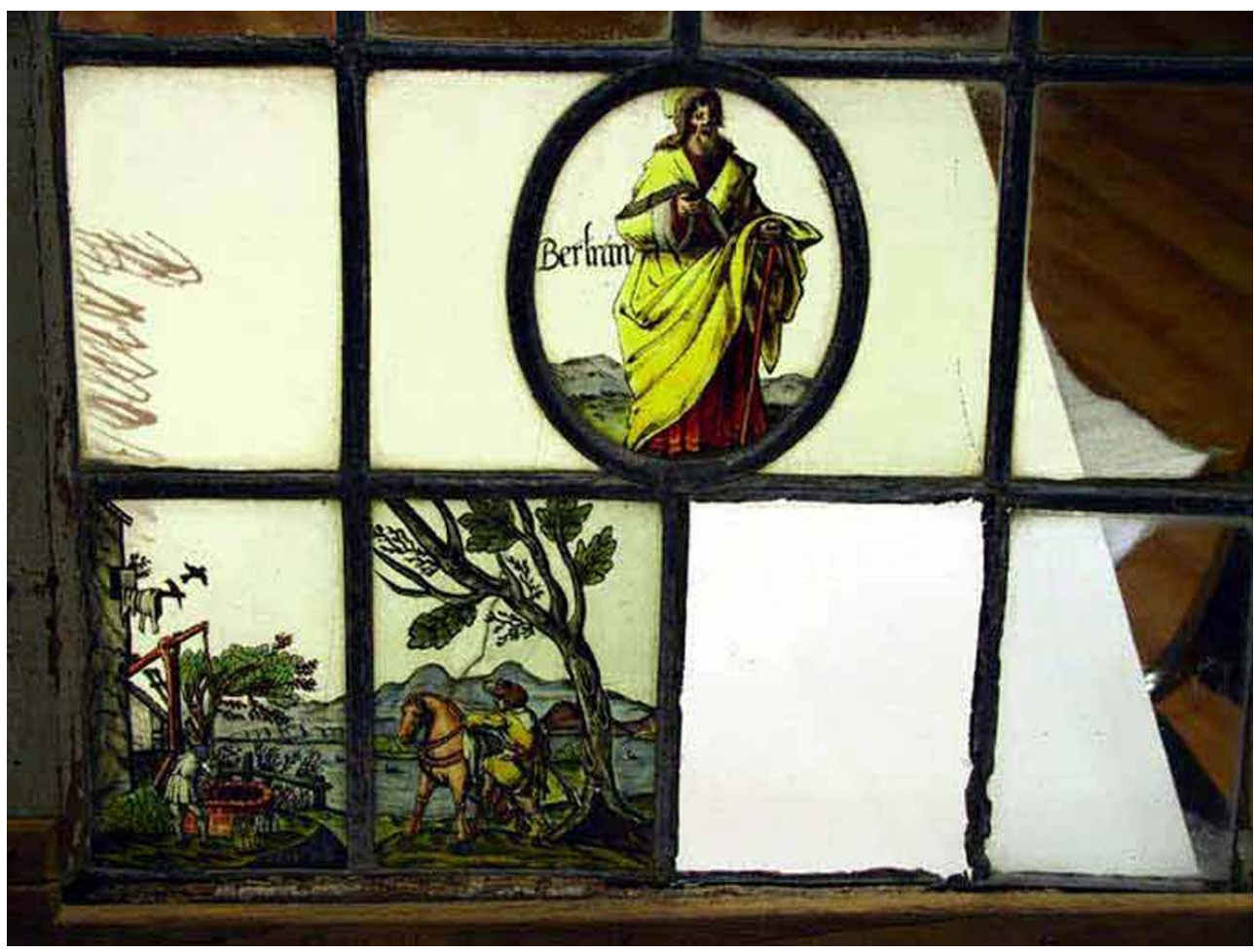

Musée du Château Ramezay, Montréal, détail : un paysan au puits et un cavalier enfourchant sa monture

(c) R. Sanfaçon, 2002

3 Deux des pièces peintes qui formaient la base du panneau de Montréal ont disparu mais les petites scènes de genre qui subsistent, un paysan au puits et un cavalier montant en selle, suffisent à prouver que tout l'ensemble était de même venue, non d'un grand artiste mais d'une qualité charmante. 


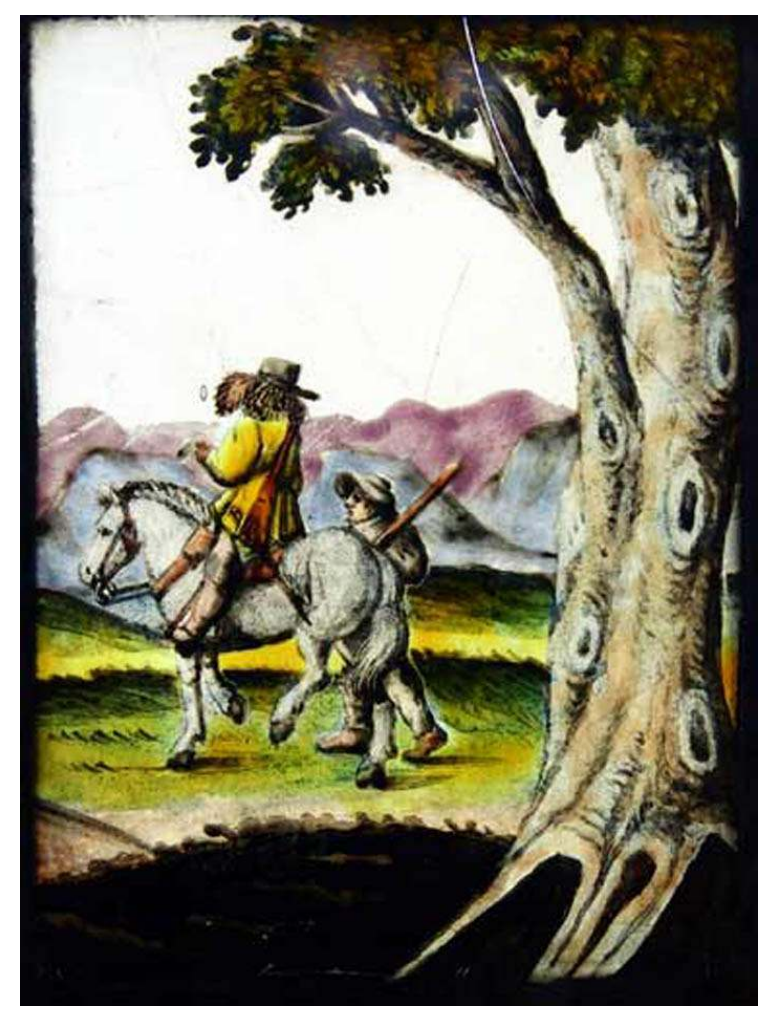

Musée du Séminaire de Sherbrooke, détail : un cavalier partant pour la chasse et son valet (c) R. Sanfaçon, 2002

Ces représentations évoquent irrésistiblement de nombreux dessins flamands et hollandais du XVII ${ }^{e}$ siècle, ceux d'Alexandre Keirincx pour ses arbres tourmentés, de Jan Van de Velde ou Louis de Vadder pour leurs pittoresques scènes rurales. On peut encore citer un dessin de Rembrandt, le «cavalier dans un paysage » du musée de l'Ermitage à Saint-Petersbourg ${ }^{5}$, qui n'est pas sans rapport avec le noble chasseur conversant avec son valet du vitrail de Sherbrooke. Il est fort probable que le peintre verrier qui reçut la commande des vitraux de Limoëlou, installé à Saint-Malo si ce n'est à Rennes, avait en sa possession de tels dessins ou les gravures qui ont pu en être tirées. Il a d'ailleurs vraisemblablement procédé à des collages à partir de divers modèles : chaque pièce porte un détail formant en soi un tableautin indépendant, et la facture plus fine de l'un, plus lourde de son voisin, laisse transparaître la pluralité des sources utilisées. Ces peintures sont exécutées en grisaille et jaune d'argent avec une forte proportion d'émaux translucides, vert, bleuté et rouge, cette dernière teinte étant curieusement utilisée pour modeler les visages des deux saints. Ces techniques, devenues familières à nombre de praticiens à partir du règne de Louis XIII, confirment, comme les costumes des figurines, une datation voisine de 1650 .

5 S'ils ont bien orné son manoir, Jacques Cartier ne put voir ces vitraux puisqu'ils sont contemporains de ceux qui occupaient la demeure près d'un siècle après lui. C'est cependant parce que le navigateur était réputé en avoir été le commanditaire qu'ils échappèrent à la destruction. Leur histoire récente explique bien la conservation, si rare, de ces " panneaux entiers » - et non d'éléments isolés, plus couramment parvenus jusqu'à nous. A l'occasion d'un pèlerinage à la maison de Jacques Cartier en 1879, l'avocat 
canadien Jérôme Adolphe Chycoine, qui fut plus tard député et maire de Sherbrooke, se fit donner par le sieur de Tarouilly, alors propriétaire de Limoëlou, le panneau qu'il devait lui-même offrir dès 1883 au musée de sa ville. L'avocat reçut l'oeuvre accompagnée, en guise de certificat d'authenticité, d'une lettre du donateur datée du 14 juin $1882^{6}$. Celle-ci documente la dépose des vitraux : pour le confort de ses fermiers, le propriétaire des lieux avait dû faire remplacer en 1855 le vitrage de la croisée de la grande salle du manoir " vu son état de vétusté » mais eut le souci de conserver « les vieux matériaux ». L'ancien fenestrage resté sans emploi se trouva donc remisé dans une dépendance du manoir jusqu'au moment où les visiteurs canadiens manifestèrent leur intérêt. Le second panneau suivit bientôt le premier Outre-Atlantique, à son tour offert comme relique ; confié par la fille du propriétaire à la duchesse de Luynes qui l'achemina au Canada en 1886, il demeura dans le domaine privé avant d'entrer dans les collections du musée du Château Ramezay en 1950.

Figure 4

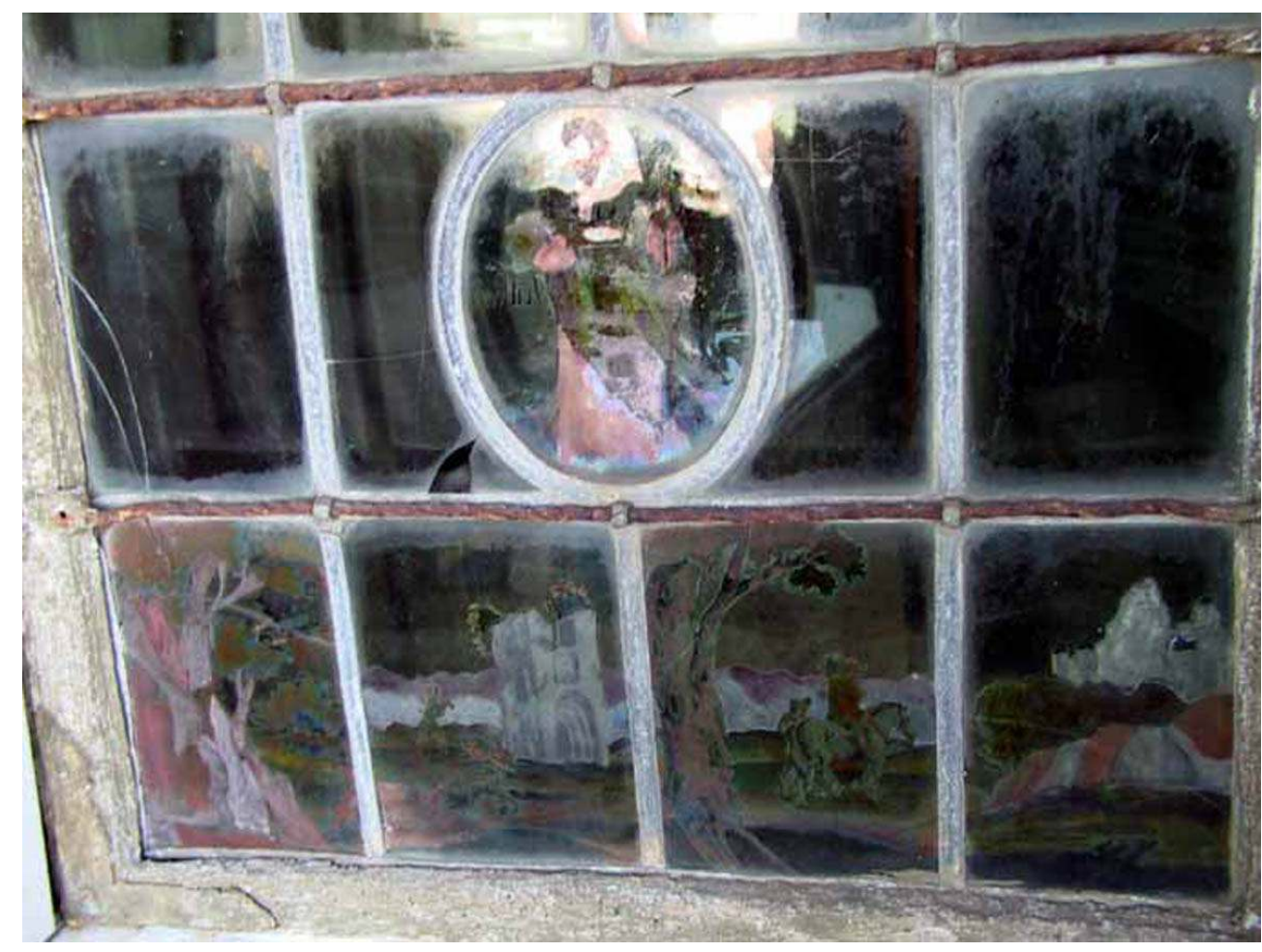

Musée du Séminaire de Sherbrooke : face externe du panneau, vergettes

(c) R. Sanfaçon, 2002

6 Les deux châssis entraient bien dans la composition de la même croisée, la salle du manoir étant du reste éclairée d'une seule baie en plus d'une porte-fenêtre moderne. On observe que tous deux ont conservé leurs vergettes de fer torsadé fixées à l'extérieur aux plombs horizontaux, et, en face interne du bâti de bois, des pièces de métal articulées, poignées ou pièces de loqueteaux indiquant qu'il s'agissait de vantaux mobiles. 
Figure 5

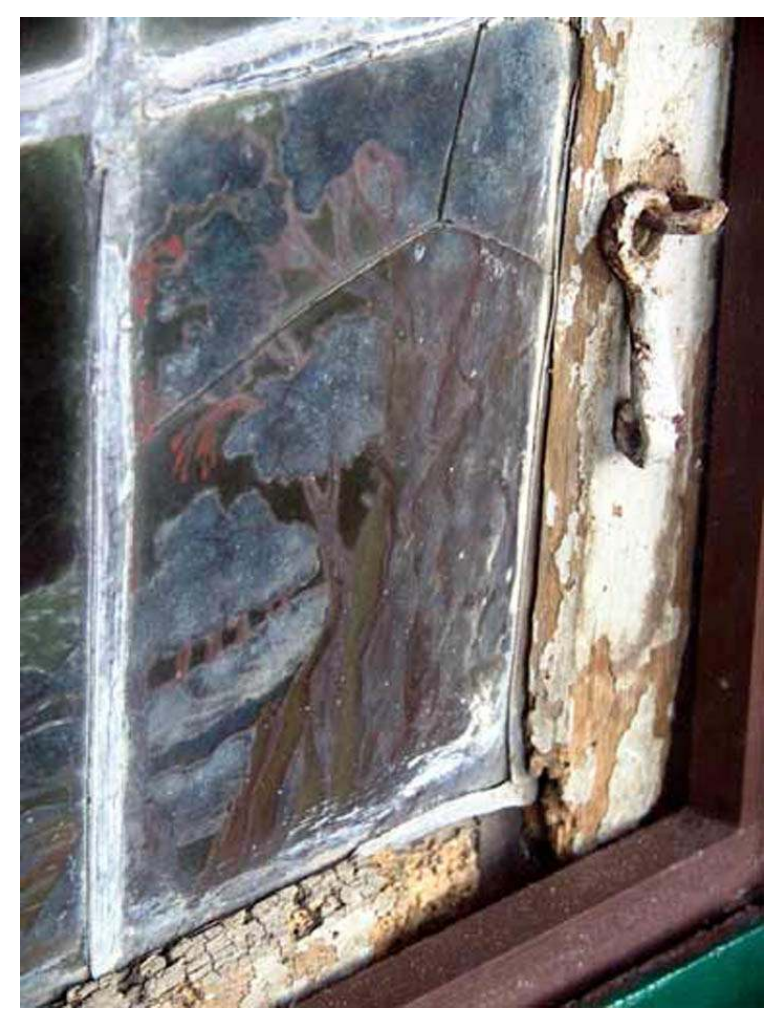

Musée du Séminaire de Sherbrooke : détail de l'une des pièces de serrurerie

(c) R. Sanfaçon, 2002

7 Sur le panneau de Sherbrooke deux de ces tirettes sont superposées du même côté de la menuiserie, celui de Montréal ne gardant que celle du haut, mais sur le montant opposé. Ces petites pièces de serrurerie permettent de se figurer la place relative qu'avaient initialement les deux vantaux : ils étaient montés de part et d'autre du meneau central, en vis-à-vis? ${ }^{7}$ Les attaches subsistantes, évidemment liées au mode de clôture de la fenêtre, servaient soit à maintenir les vantaux fermés à l'aide des pièces complémentaires ancrées au long du meneau, soit à la fixation de volets intérieurs qui se rabattaient devant les vitrages ${ }^{8}$. La salle du manoir prenait le jour, on l'a dit, par une fenêtre unique. Or Banéat a mentionné qu'il existait au XIXe siècle trois panneaux supplémentaires; ceux-ci n'ont pu faire partie du même ensemble, à moins que la baie n'ait compris trois niveaux de châssis superposés. Dans cette hypothèse, on imaginerait volontiers que les panneaux perdus, faits de simple vitrerie, en occupaient les registres hauts, les petits sujets peints, destinés à la contemplation, étant placés en partie basse. Une vue du manoir antérieure aux modifications qu'il a subies au XIXe siècle pourrait seule le confirmer. 


\section{NOTES}

1. Cette collection d'ouvrages créée en 1978 s'inscrit dans la série internationale du Corpus Vitrearum ; elle présente l'inventaire exhaustif des vitraux français antérieurs à la Révolution. Elle est coéditée par Monum, Editions du Patrimoine et CNRS-Editions.

2. Baneat, (Paul). Le département d'Ille-et-Vilaine, Histoire, archéologie, monuments. Rennes, t. III, 1929. P. 53.

3. Qu'avec lui soient remerciés $M$. Claude Thibault, du musée du Québec, qui a rapidement localisé les vitraux et constitué un premier dossier documentaire, M. Serge Gauthier, conservateur du musée de Sherbrooke, Mme Marie Chapdeleine son assistante, ainsi que M. JeanFrançois Royal, archiviste du musée de Montréal.

4. Le château de Montgothier (Manche) a conservé quelques panneaux de son vitrage original du $\mathrm{XVII}^{\mathrm{e}}$ siècle; ils sont comparables à ceux qui nous occupent par leur échelle et le calibre rectangulaire des pièces mises en plomb, mais ce sont de simples carreaux sans décor ; Album du Centre de Recherche sur les Monuments Historiques, Fenêtres et volets. t. 7, Paris, s. d. (vers 1975), D 10661.

5. Ermitage, inv. 5316 ; reproduit dans le catalogue d'exposition Dessins flamands et hollandais du XVII ${ }^{e}$ siècle du musée de l'ermitage, Leningrad, et du Musée Pouchkine, Moscou. Paris, Institut néerlandais, $1973, \mathrm{n}^{\circ} 77$ et p. 51-52.

6. Archives du musée du Séminaire de Sherbrooke.

7. Le panneau de Sherbrooke occupait la travée de gauche, celui de Montréal celle de droite.

8. Pour alimenter la réflexion, de nombreux relevés de fenêtres à châssis mobiles doublées de volets figurent dans les albums de la collection mentionnée à la note 4. Voir en particulier : Album du centre de recherche sur les monuments historiques, Menuiseries de fenêtres, première moitié du XVI ${ }^{\mathrm{e}}$ siècle (avec introduction de D. Bontemps). Paris, 1992.

\section{INDEX}

Mots-clés : vitrail, verrière, iconographie religieuse

Keywords : ornementation

\section{AUTEUR}

\section{FRANÇOISE GATOUILLAT}

Ingénieur d'études, cellule vitrail, Sous-direction des études, de la documentation et de

l'Inventaire. francoise.gatouillat@culture.gouv.fr 